\title{
Impact of Pulse Width on the Sensitivity and Range of a Raman-based Distributed Fiber- optic Temperature Sensor
}

\author{
Marianne S. P. e Silva ${ }^{1}$, Henrique P. Alves ${ }^{1}$, Jehan F. do Nascimento ${ }^{2}$, Joaquim F. Martins-Filho ${ }^{1}$ \\ ${ }^{I}$ Department of Electronics and Systems, Federal University of Pernambuco. Recife, Brazil \\ marianne_stely@hotmail.com,jfmf@ufpe.br \\ ${ }^{2}$ Interdisciplinary Nucleus for Exact and Natural Sciences, Federal University of Pernambuco. Caruaru, Brazil. \\ Jehan.nascimento@ufpe.br
}

\begin{abstract}
This work presents the operation of a spontaneous Raman scattering-based distributed fiber-optic temperature sensor using a commercial OTDR and a standard EDFA optical amplification in a simple and economic scheme. We present both theoretical and experimental results regarding the sensor's sensitivity and performance in seven different configurations, using OTDR pulses of $100 \mathrm{~ns}$ to $4000 \mathrm{~ns}$ for two detection regions, in the beginning and ending sections of a $27 \mathrm{~km}$ standard single-mode fiber sensor link. The results reveal the trade-off between sensor sensitivity, resolution and range, regarding the use of different OTDR pulsewidths.

Index Terms - Raman Scattering, Optical Fiber, OTDR, temperature sensor, optical sensor.
\end{abstract}

\section{INTRODUCTION}

Fiber-optic sensors have been studied for more than 40 years now. Since the beginning, various optical sensors have been proposed to monitor a wide variety of physical parameters [1]-[3]. In distributed fiber-optic sensors, the profile of a physical parameter can be gauged throughout the fiber. The features of distributed intrinsic sensors make them suitable for a plethora of applications that require monitoring a certain parameter continuously or in a large number of sections [2].

Raman-based distributed fiber-optic temperature sensors (R-DTS) have been proposed for a number of applications, such as temperature monitoring in high-voltage power lines, prevention and detection of fires in oil wells, aeronautical and space applications, among others [2], [3]. Optical fiber dielectric material also enables the use of R-DTSs in hostile environments, such as explosive or corrosive atmospheres, nuclear plants, and environments with high electromagnetic interference. Furthermore, these sensors may be used to monitor temperatures in a difficult-to-reach remote location (dozens of kilometers away) [1].

In general, temperature detection based on Raman scattering is in turn based on optical timedomain reflectometry (OTDR), and the distribution of temperature is obtained by measuring the intensity of the backscattered Stokes and anti-Stokes signals originating from the Raman scattering. However, the low intensity of the backscattered anti-Stokes emission poses some limitations on sensitivity and reach. For this reason, amplification modules are inserted into the project of these Brazilian Microwave and Optoelectronics Society-SBMO received 28 Sept 2018; for review 6 Nov 2018; accepted 20 Nov 2018 Brazilian Society of Electromagnetism-SBMag () 2018 SBMO/SBMag (cc) BY ISSN 2179-1074 
sensors systems [4], [5].

In the last decades, many studies were published on techniques and measurement protocols, as a means to foster R-DTS systems, by improving parameters such as operating range, sensitivity, spatial resolution, costs, etc. [6]-[9]. Viable and economic R-DTSs, operating at distances of $40 \mathrm{~km}$, have already been analyzed and tested [10]. One example of high-performance temperature sensor is the RDTS scheme based on coded OTDR along with the discrete Raman amplification discussed in [10]. Using pulses of $100 \mathrm{~ns}$, this system demonstrated the increase of the distance range by about $25 \mathrm{~km}$ when compared to the use of conventional OTDR, whose range is of approximately $15 \mathrm{~km}$ at a resolution of $5 \mathrm{~K}$ in temperature [10]. An R-DTS using a commercial optical time-domain reflectometer (OTDR) and a gain-controlled Erbium-doped fiber amplifier (EDFA) was proposed in [11]. This work illustrated the viability of implementation and the potential of good-performance RDTSs using commercially available OTDRs. These works explored the use of low-noise signal codification and amplification techniques in order to improve the sensitivity and performance of RDTSs, but there are no reports in the literature that evaluate the impact of OTDR pulse width in the sensitivity, range, and in the general performance of the sensor.

In this work, we present results from a R-DTS implemented using a commercial OTDR and a standard EDFA amplifier so that we can evaluate the implications of different pulse widths in the measurement of temperatures at short and long distances in the optical fiber.

\section{EXPERIMENTAL SETUP}

Fig. 1 illustrates the experimental setup employed to implement the Raman-based distributed temperature sensor using commercial OTDR and standard EDFA.

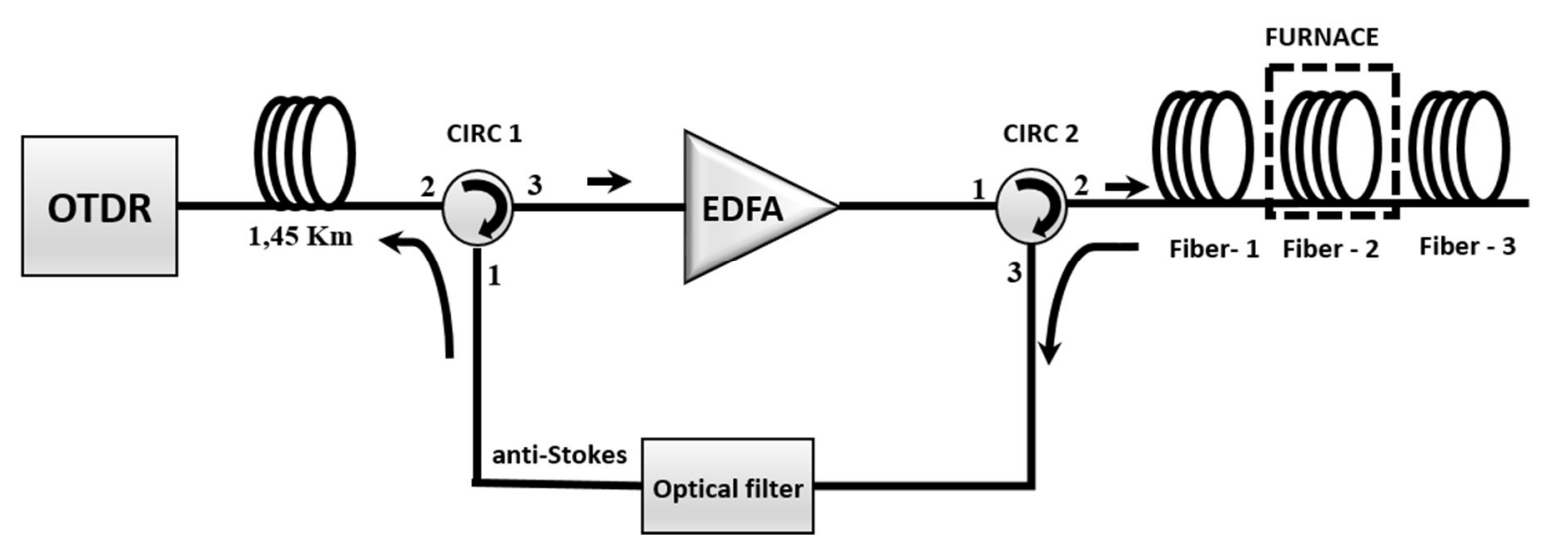

Fig. 1. Experimental setup used to implement R-DTS using commercial OTDR.

In the system of Fig. 1, the OTDR, operating at $1550 \mathrm{~nm}$, emits periodic light pulses with predefined temporal width. Such pulses are amplified by an EDFA and injected into the sensor link by means of an optical circulator. The OTDR used is an Anritsu (Model MT9083C2), with maximum 
optical power of $0.15 \mathrm{~W}, 3 \mathrm{~ns}$ pulses at $20 \mu \mathrm{s}$ and dynamic range of $45 \mathrm{~dB} @ 20 \mu \mathrm{s}$ and 25 dB@ 100 ns. The EDFA was implemented with 10 m of Erbium-doped fiber (type M3 1480/125), and a pumping laser at $980 \mathrm{~nm}$. In addition, we configured the EDFA to produce $18 \mathrm{dBm}$ of output power when the pumping power is $36.5 \mathrm{~mW}$. The amplified OTDR pulses, when propagated throughout the sensing optical fiber, stimulate the occurrence of Spontaneous Raman Scattering. A spool of standard single-mode fiber, $1.45 \mathrm{~km}$ in length, was inserted into the entry point of the system in order to avoid the dead zone of the OTDR. Two 3-port optical circulators (CIRC 1, CIRC 2) compose the feedforward and feedback circuit. The circulators block signals reflected between them, and they direct the backscattered signals back to the OTDR. The backscattered signals are, in turn, filtered by an optical bandpass filter (1400-1480 nm), which is used to separate the anti-Stokes band and to avoid the interference of the amplified spontaneous emission (ASE) in the detected signal. The OTDR curves of the detected signals were stored for processing. The optical fiber sensor link was composed by three spools of standard single-mode fiber (Fiber-1, Fiber-2 and Fiber-3) with lengths of $24.6398 \mathrm{~km}, 0.6269 \mathrm{~km}$ and $1.8897 \mathrm{~km}$, and attenuation coefficients at $1550 \mathrm{~nm}$ of $\sim 0.19 \mathrm{~dB} / \mathrm{km}$. The Fiber-2 spool $(0.6269 \mathrm{~km})$ was inserted in a muffle furnace of controlled temperature (FURNACE). The values of Fiber-1 and Fiber-3 lengths varied throughout the measurements and they are kept at room temperature. The operation of the sensor was experimentally evaluated within the temperature range of $30^{\circ} \mathrm{C}$ to $100^{\circ} \mathrm{C}$.

The combination of a commercial OTDR and a standard EDFA, in the proposed scheme, was considered to be advantageous, since both consist in compact systems, and the EDFA is a lower cost and lower power consumption solution, compared to other types of amplifiers. Besides, the utilization of a commercial OTDR in the R-DTS presents practical advantages, as it allows for the configuration of the emitting/detecting system for a variety of parameters and the analysis of the sensor's important aspects, namely sensitivity and range, as a function of these parameters. Based on this, the R-DTS of Fig. 1 was tested for OTDR pulses of different widths with the aim of enabling a sensor sensitivity analysis for different heated regions along the fiber. To accomplish such goal, we made use of pulses with temporal widths ranging from $100 \mathrm{~ns}$ to $4000 \mathrm{~ns}$. Table I shows the information on the temporal width of OTDR pulses used in some R-DTS systems reported in the literature. We observe that the results found in the literature are for pulse widths ranging from 10 to $100 \mathrm{~ns}$.

TABLE I. TEMPORAL PULSE WIDTH $(\Delta \mathrm{T})$ IN R-DTS SYSTEMS EMPLOYED BY SOME AUTHORS

\begin{tabular}{cc}
\hline Reference & $\Delta \mathbf{t ~ ( n s ) ~}$ \\
\hline Dakin et al., (1985) [12] & 15 \\
Hartog et al.,(1985) [13] & 40 \\
Park et al., (2006) [14] & 100 \\
Blognini et al., (2007) [10] & 100 \\
Signorini et al., (2010) [15] & 10 \\
Hwang et al., (2010) [16] & 50 \\
Soto et al., (2011) [17] & 10 \\
Bassan et al., (2015) [5] & 100 \\
Bassan et al., (2016) [11] & 100 \\
\hline
\end{tabular}




\section{THEORETICAL MODEL FOR DISTRIBUTED TEMPERATURE SENSOR}

This section reviews a theoretical model for a Raman-based distributed fiber-optic temperature sensor [18]. The Bose-Einstein distributions for the Stokes (S) and anti-Stokes (AS) modes are given by the equations

$$
\rho_{S}=\frac{1}{1-e^{\frac{\Delta E}{k T}}}
$$

and

$$
\rho_{A S}=\frac{e^{\frac{\Delta E}{k T}}}{1-e^{\frac{\Delta E}{k T}}},
$$

where $k$ is Boltzmann's constant, $T$ is the temperature throughout the optical fiber, $\Delta E=h \Delta v$ is the difference between the energy levels of the initial and final states [19], typically $50 \mathrm{meV}$ for $\mathrm{SiO}_{2}$ [18], $h$ is Planck's constant and $\Delta v$ is the frequency shift of the Stokes and anti-Stokes modes. From equations (1) and (2), the dependence of both modes on the temperature is noteworthy [19].

The backscattered optical powers associated with the Stokes and anti-Stokes modes are described by

$$
P_{S}(z)=P_{0} \rho_{S} \Gamma_{S} \Delta z e^{-\left(\alpha_{P 0}+\alpha_{P S}\right) z}
$$

and

$$
P_{A S}(z)=P_{0} \rho_{A S} \Gamma_{A S} \Delta z e^{-\left(\alpha_{P 0}+\alpha_{P A S}\right) z},
$$

where $P_{0}$ is the pumping power, $\rho_{S}$ and $\rho_{A S}$ are the Bose-Einstein distributions for the Stokes and antiStokes modes, respectively, $\Gamma_{S}$ and $\Gamma_{A S}$ are the capture coefficients of the Stokes and anti-Stokes modes, respectively, $\alpha_{P O}$ is the attenuation coefficient of the optical fiber in the pumping wavelength, $\alpha_{P S}$ and $\alpha_{P A S}$ are the attenuation coefficients of the Stokes and anti-Stokes propagation modes, respectively [19], $z$ is the variable associated with the distance along the optical fiber. $\Delta z=c \Delta t / n$ is the spatial resolution, which depends of the pulse width $\Delta t$, the speed of light $c$ and the refractive index of the nucleus of the fiber $n$ [19]. Table II presents the values of the parameters involved in the model.

Based on the set of equations presented (1)-(4), we implemented, in Matlab ${ }^{\odot}$, the model for the distributed temperature sensor. This model takes into consideration the actual parameters of the experiment described in Section II, such as length of the fiber under heating and the complete link, pulse width employed by the OTDR and optical power. Because the computational modeling allows for more simulation possibilities, the temperature range was not limited to the one used in the experiment. 
TABle II. Parameters VAlues for Theoretical Model [19].

\begin{tabular}{ccc}
\hline Parameter & Value & Unit \\
\hline$\alpha_{P O}$ & 0.2 & $d B / \mathrm{km}$ \\
$\alpha_{P S}$ & 0.25 & $d B / \mathrm{km}$ \\
$\alpha_{P A S}$ & 0.22 & $d B / \mathrm{km}$ \\
$\Gamma_{S}$ & $3.04 \times 10^{-10}$ & $\mathrm{~m}^{-1}$ \\
$\Gamma_{A S}$ & $4 \times 10^{-10}$ & $\mathrm{~m}^{-1}$ \\
$P_{0}$ & 150 & $\mathrm{~mW}$ \\
$c$ & $3 \times 10^{8}$ & $\mathrm{~m} / \mathrm{s}$ \\
$\Delta t$ & 100 & $\mathrm{~ns}$ \\
$n$ & 1.4443 & - \\
\hline
\end{tabular}

\section{RESULTS}

The temperature values were obtained based on measurements of OTDR traces from the sensor link using a commercial OTDR along with an EDFA for six different conditions, which combine the width of the emitted pulses and the analyzed region under heating. The backscattered anti-Stokes signals were measure in two different temperature situations: in the first one, all fiber spools were kept at room temperature; in the second situation, the Fiber-2 spool underwent controlled heating at different temperatures (up to $100{ }^{\circ} \mathrm{C}$ ) by means of a muffle furnace (see Fig. 1). The heating temperature was monitored by a thermostat in the interior of the muffle furnace heating chamber. The OTDR equipment provides light intensity curves of the backscattered anti-Stokes signals as a function of the distance in fiber, for different temperatures.

The temperature profile of the distributed detection system could be obtained solely from the antiStokes traces by means of the calibration of the system with a known temperature, a reference measurement and data processing. Using the previously configured OTDR, we obtained intensity curves with $7.5 \mathrm{~min}$ of measurement averages in the OTDR equipment, which amounts to up to 419 traces, the density of 25001 data points for different temperature values adjusted in the furnace, always ranging from $30{ }^{\circ} \mathrm{C}$ to $100^{\circ} \mathrm{C}$.

Fig. 2 exhibits the OTDR curves for the intensity of the anti-Stokes signal as a function of the distance, for pulses of $100 \mathrm{~ns}$ and $400 \mathrm{~ns}$, with the heated Fiber-2 spool at the position $3.4 \mathrm{~km}$ in the link, for different temperatures. Fig. 2 (a) shows the anti-Stokes traces for OTDR pulses of $100 \mathrm{~ns}$ for different temperatures between $33^{\circ} \mathrm{C}$ and $100{ }^{\circ} \mathrm{C}$, and Fig. 2 (b) showcases the traces obtained by the use of $4000 \mathrm{~ns}$ OTDR pulses for temperatures ranging from 45 to $100{ }^{\circ} \mathrm{C}$. Note that, in both cases, it is possible to detect the heating regions in the sensor link and distinguish $5{ }^{\circ} \mathrm{C}$ temperature variations. Nonetheless, a more accurate analysis leads to the conclusion that the sensor operating at $100 \mathrm{~ns}$ shows slightly higher sensitivity in relation to the same system using $4000 \mathrm{~ns}$ pulses $\left(0.0169 \mathrm{~dB} /{ }^{\circ} \mathrm{C} @\right.$ $\left.100 \mathrm{~ns}, 0.0134 \mathrm{~dB} /{ }^{\circ} \mathrm{C} @ 400 \mathrm{~ns}\right)$. The spatial resolution of OTDR depends on the emitted pulse width in a way that shorter pulses result in better spatial resolution. As a result, the shape of the anti-Stokes signals on Fig. 2 (a) and (b) are different. 


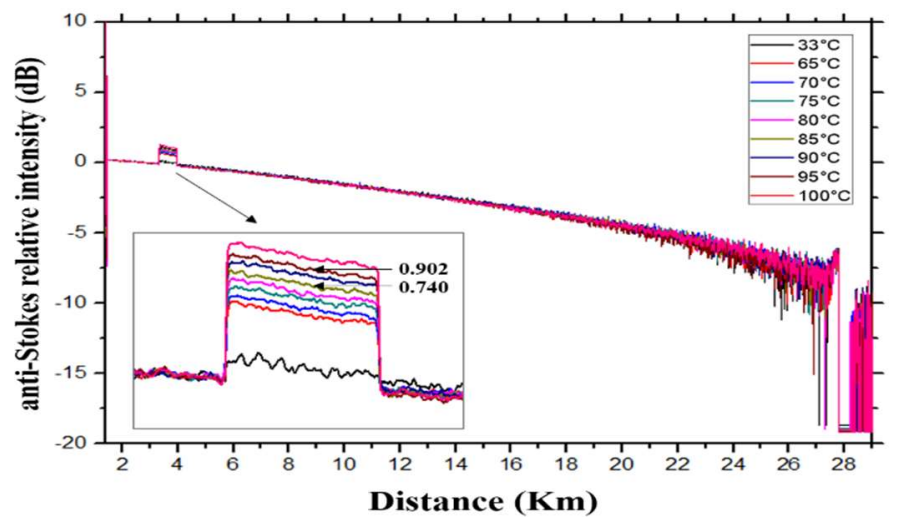

(a)

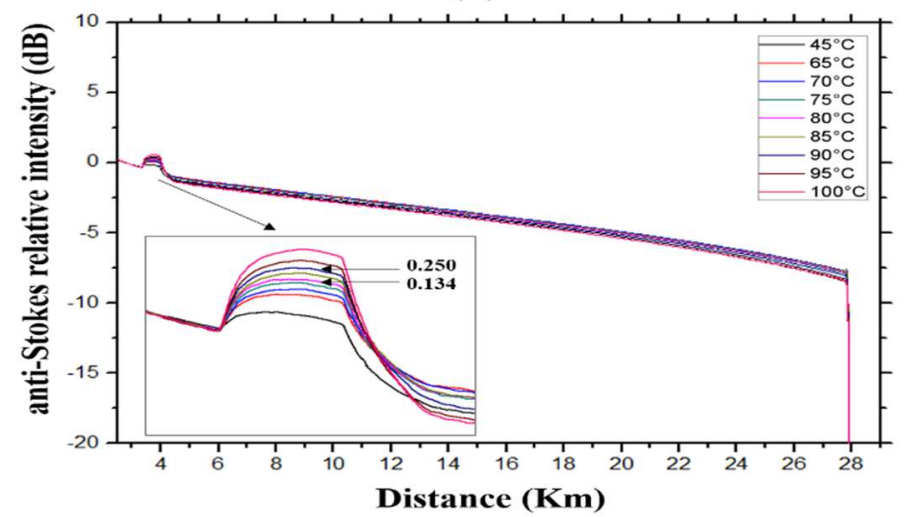

(b)

Fig. 2. OTDR curves of the backscattered Raman anti-Stokes signals with the heated Fiber-2 spool at the position 3.4 $\mathrm{km}$, for OTDR pulses of (a) $100 \mathrm{~ns}$ and (b) $4000 \mathrm{~ns}$.

Fig. 3 shows the intensity curves for the backscattered Raman anti-Stokes signal as a function of the distance, measured experimentally and obtained by means of the theoretical model, in the same conditions as those of Fig. 2 (a), for heating temperatures of $33{ }^{\circ} \mathrm{C}, 65^{\circ} \mathrm{C}$ and $100{ }^{\circ} \mathrm{C}$. These results show that, in the heating region, the intensity of the theoretically obtained signals differ only slightly from those obtained experimentally. This small difference in intensity is due to the limitations of the theoretical model, as it does not take into account additional signal losses and noise, neither the amplification of the signal carried out in the experimental step. For this reason, the theoretical curves undergo an adjustment of $1.37 \mathrm{~dB}$. In spite of this, the theoretical and experimental results present a considerable degree of equivalence.

The spatial distribution of temperature (the temperature along the fiber) was determined by means of system calibration lines, i.e., lines from which the temperature value can be determined from the intensity of the measured signal. These lines are determined by the linear fitting of the points obtained experimentally. In this process, the fitting of the points obtained experimentally with calibration curves gives the accuracy of the measurement, which was above $98 \%$ for OTDR pulses of $100 \mathrm{~ns}$ and 4000 ns. 


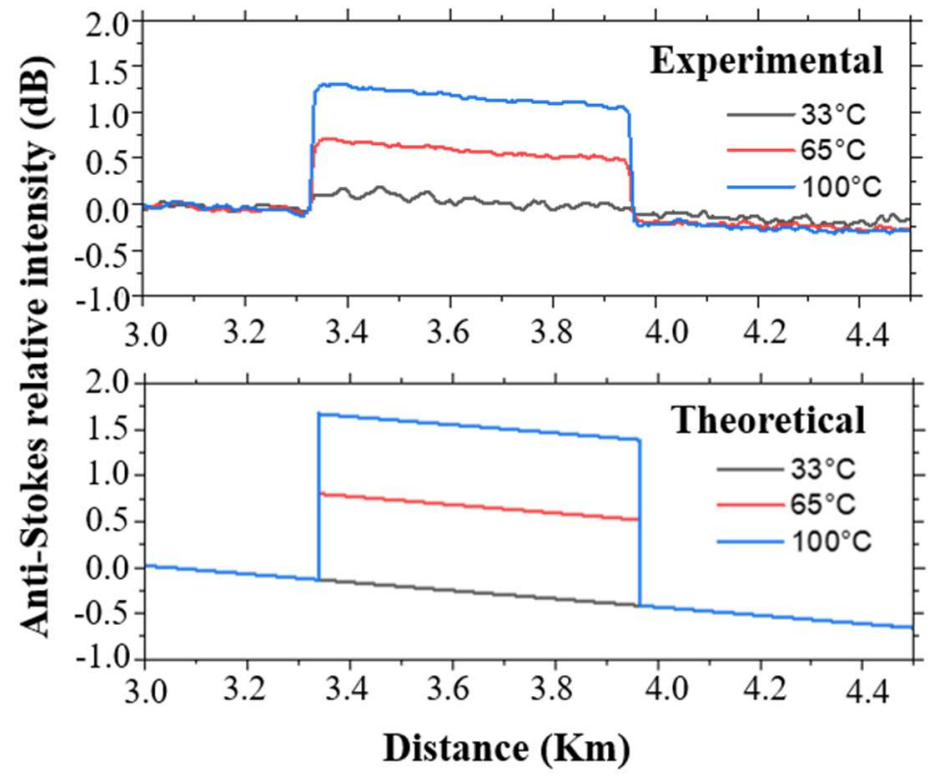

Fig. 3. Intensity curves of the backscattered Raman anti-Stokes signal for $627 \mathrm{~m}$ of optical fiber heated at the mark of $3,4 \mathrm{~km}$ of the sensor link, obtained experimentally and from the theoretical modeling.

The spatial distribution of temperatures for the results of Fig. 2 are illustrated in Fig. 4. Fig. 4 (a) and (b) show the temperature curves as a function of the distance for pulses of $100 \mathrm{~ns}$ for different temperatures. In this case, it is possible to detect temperature fluctuations in the heated fiber spool (Fibra-2) at the mark $3.4 \mathrm{~km}$ with a resolution of $5{ }^{\circ} \mathrm{C}$ and average precision of $2{ }^{\circ} \mathrm{C}$.

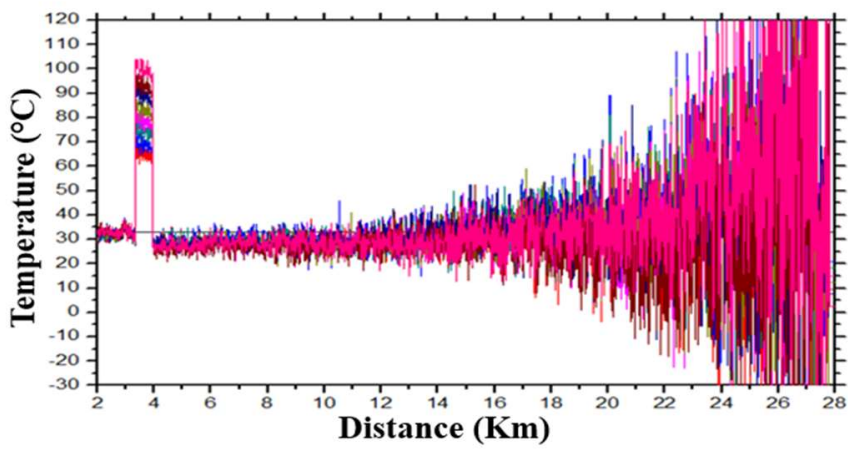

(a)

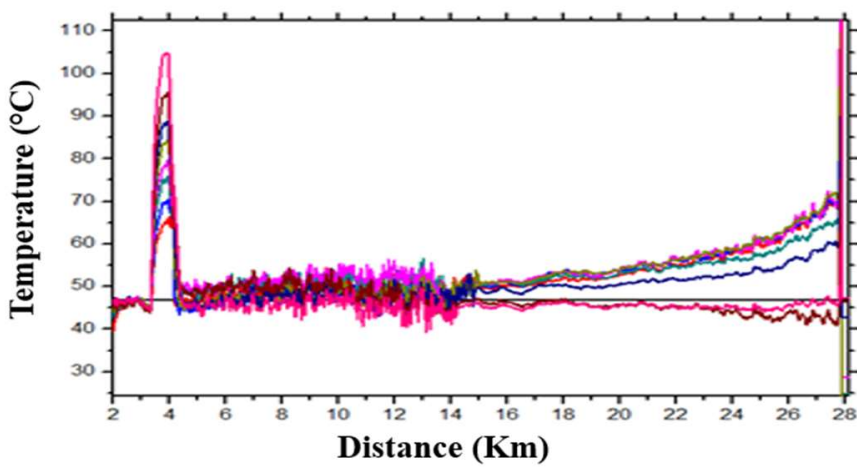

(c)

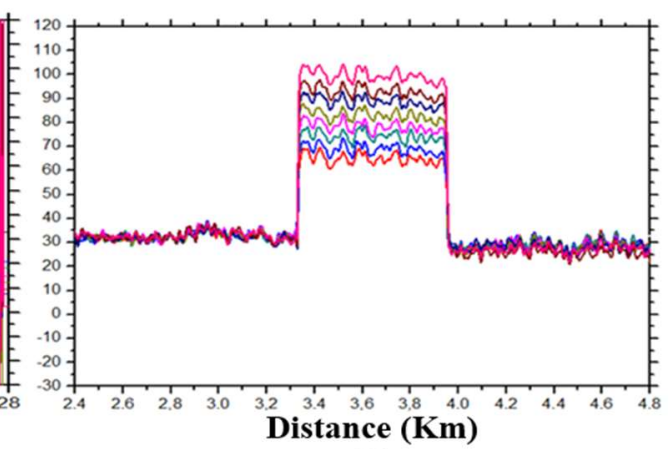

(b)

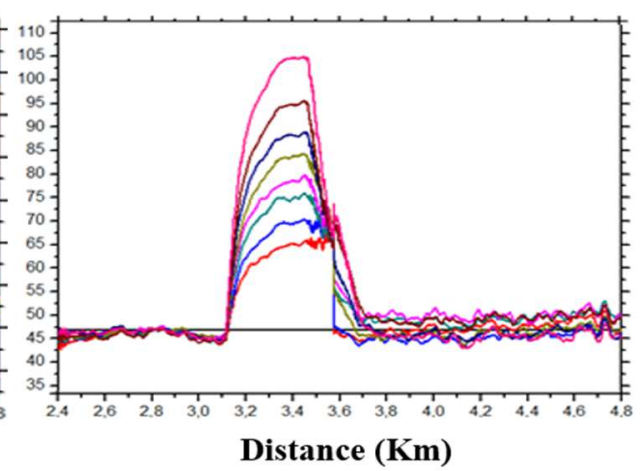

(d)

Fig. 4. Temperature distribution as a function of fiber length and temperature spikes measured using $627 \mathrm{~m}$ of heated fiber at the mark of $3.4 \mathrm{~km}$, with OTDR pulses of $100 \mathrm{~ns}$ ( $\mathrm{a}$ and b) and $4000 \mathrm{~ns}$ (c and d). 
Fig 4. (a) also allows the conclusion that the range of the sensor is limited by the distance of approximately $15 \mathrm{~km}$, in this case. For longer distances, the curves are too noisy and less practical for the identification of small variations in temperature. The effect of the increase in pulse width can be visualized in Fig. 4 (c) and (d). It is evident from Fig. 4 (c) that the temperature distribution obtained using pulses of $4000 \mathrm{~ns}$ results in an increase in the distance of measurement by about $12 \mathrm{~km}$ in comparison to the same system operating at the pulse width of $100 \mathrm{~ns}$. When $4000 \mathrm{~ns}$ pulses were employed, we obtained a temperature resolution of $5{ }^{\circ} \mathrm{C}$ and average precision of $5{ }^{\circ} \mathrm{C}$. This means that, even though the temperature measurement was more accurate when using pulses of $100 \mathrm{~ns}$, the operation of the sensor for such pulse width renders unviable the detection of temperature in the final kilometers of the sensor link. On the other hand, remote regions can be monitored by making use of 4000 ns pulses.

We also evaluated the sensitivity of the sensor in a heating region located near the end of the sensor link, at about $26 \mathrm{~km}$ mark. For such, the position of the Fiber-2 spool composing the sensor link was altered so that we could maintain the same length of the fiber under temperature variations. In this scenario, Fiber-2 was allocated at the $25.4 \mathrm{~km}$ mark. Maintaining the same measurement protocol, the intensity of the backscattered Raman anti-Stokes signals was measured for four different pulse width values: 100, 1000, 2000 and 4000 ns. The experimental results of the intensity curves for the antiStokes signal throughout the length of the optical link for pulses of width between 100 and $4000 \mathrm{~ns}$ are presented in Fig. 5.

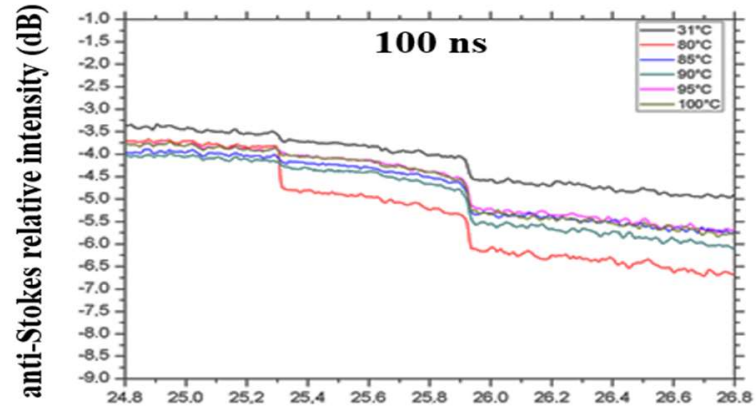

Distance (Km)

(a)

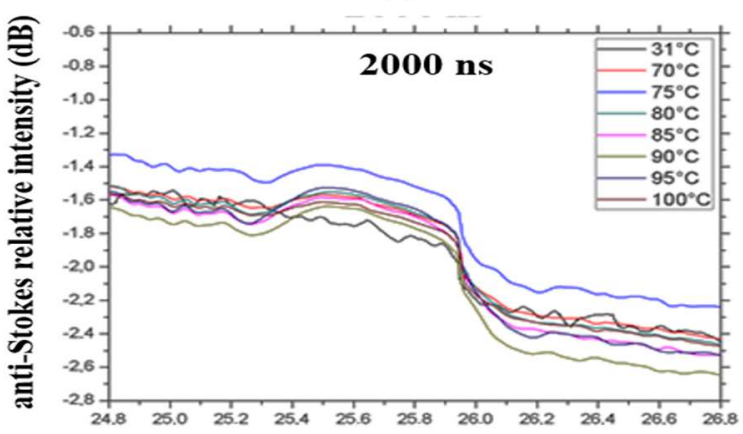

Distance (Km)

(c)

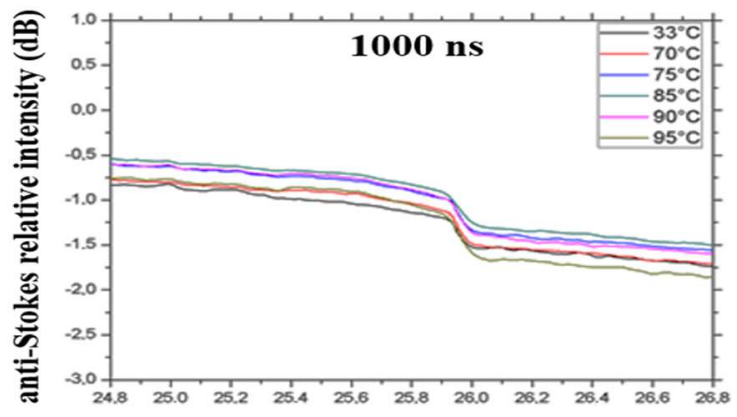

Distance (Km)

(b)

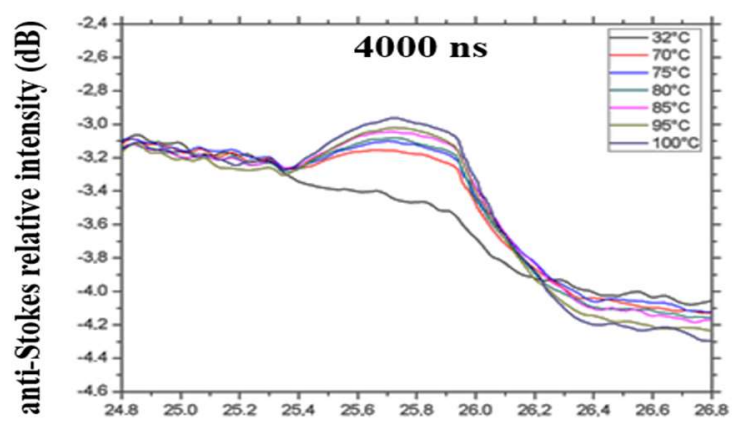

Distance (Km)

(d)

Fig. 5 OTDR curves of the backscattered Raman anti-Stokes signals with the heated Fiber-2 spool at the position $25.4 \mathrm{~km}$, for OTDR pulses of (a) $100 \mathrm{~ns}$, (b) $1000 \mathrm{~ns}$, (c) $2000 \mathrm{~ns}$ e (d) $4000 \mathrm{~ns}$. 
Fig. 5 (a) shows that it was not possible to identify consistent variations in the anti-Stokes signal intensity in relation to the heating of Fiber- 2 at the position of $25.4 \mathrm{~km}$, as the sensor has an extremely low sensitivity for this configuration $\left(\sim 10^{-5} \mathrm{~dB} /{ }^{\circ} \mathrm{C}\right)$. A similar result was obtained when pulses of 1000 ns were used, as shown in Fig. 5 (b), inhibiting the measurement of different temperatures. The increase in the intensity of the anti-Stokes signal was detectable when OTDR pulses of $2000 \mathrm{~ns}$ were emitted, as Fig. 5 (c) illustrates. Although changes in the form of the OTDR curves could be identified for the anti-Stokes signal, the increase in the intensity of the signal as a function of the temperature was insufficient to adequately distinguish variations of $70{ }^{\circ} \mathrm{C}$ in temperature. The OTDR curves for the backscattered anti-Stokes signal measured for pulses of $4000 \mathrm{~ns}$ are presented in Fig. 5 (d). In this case, the obtained curves for temperature variations ranging from $32{ }^{\circ} \mathrm{C}$ to $95{ }^{\circ} \mathrm{C}$ could be resolved with steps of $10^{\circ} \mathrm{C}$ in temperature.

The spatial distributions of the measured temperatures, for the Fiber-2 spool placed at the $25.4 \mathrm{~km}$ mark, using 4000 ns OTDR pulses, are presented in Fig. 6. This result demonstrates the operation of the sensor towards the end section of the sensor link with temperature resolution of $10{ }^{\circ} \mathrm{C}$ and sensitivity of $0.0075 \mathrm{~dB} /{ }^{\circ} \mathrm{C} @ 4000$ ns. The effect caused by the increase in pulse can also be observed in the extension of the measurement range. Fig. 6 (a) shows the range improvement of 12 $\mathrm{km}$, when compared to the results obtained using OTDR pulses of $100 \mathrm{~ns}$ (Fig. 4 a), enabling, for example, the detection of temperature variation at the distance of $26 \mathrm{~km}$.

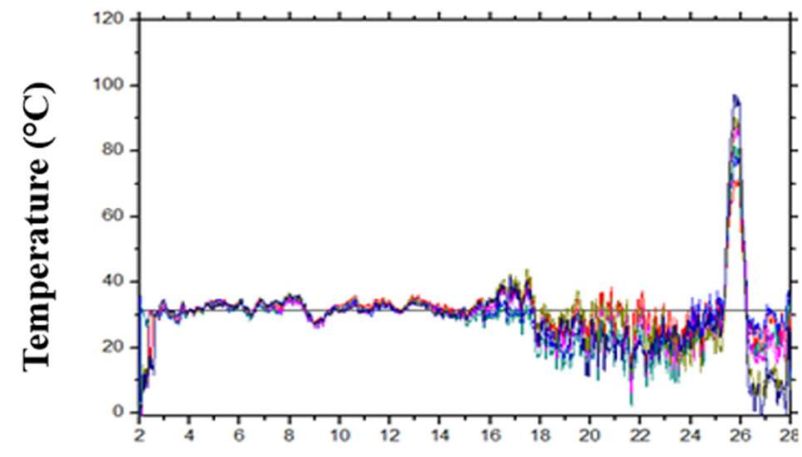

Distance (Km)

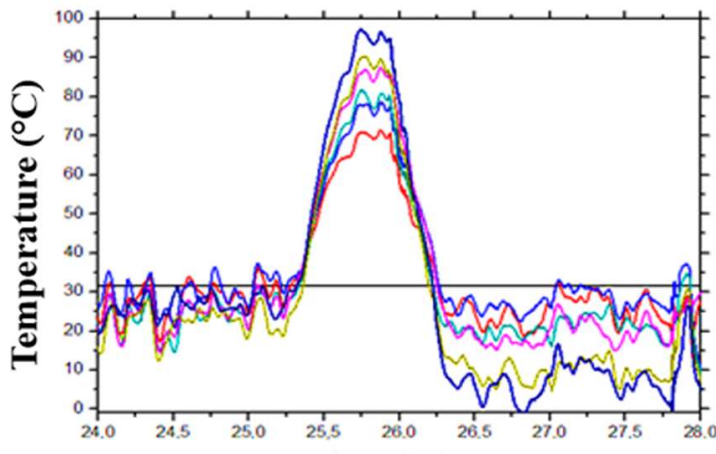

Distance (Km)

(a)

(b)

Fig. 6. Temperature distribution as a function of fiber length using $627 \mathrm{~m}$ of fiber heated at the mark of $25.4 \mathrm{~km}$, using OTDR pulses of $4000 \mathrm{~ns}$ (a) and measured temperature spikes (b).

The sensor has also been evaluated for temperature variations in a fiber of reduced length. In this scenario, $2 \mathrm{~m}$ of SMF fiber were inserted into the controlled-heat chamber (FURNACE), at the position of the Fiber-2, and subjected to temperature variations ranging from $34{ }^{\circ} \mathrm{C}$ to $100{ }^{\circ} \mathrm{C}$. At the Fiber-1 and Fiber-3 positions, the spools with $1.88 \mathrm{~km}$ and $24.4 \mathrm{~km}$ of optical fiber, respectively, were maintained. Fig. 7 shows the OTDR curves of relative intensity of the backscattered Raman antiStokes signal as a function of the distance in the sensor link for $100 \mathrm{~ns}$ pulses, maintaining the 
FIBER-2 spool, now 2 meters long, at the position of $3.4 \mathrm{~km}$ under varying temperatures. The curves depicted in Fig. 7 evidence that it is possible to identify intensity variations in the optical signal caused by temperature fluctuations at such small fiber length. Under these circumstances, $10{ }^{\circ} \mathrm{C}$ variations were identified in the heated fiber, with an average increase of $0.17 \mathrm{~dB}$ in the intensity of the measured anti-Stokes signal. This result evaluates the limitations of the sensor's spatial resolution, and presents an analysis of its performance under such conditions, since the performance is analyzed near the OTDR spatial resolution, which is of $1 \mathrm{~m}$.

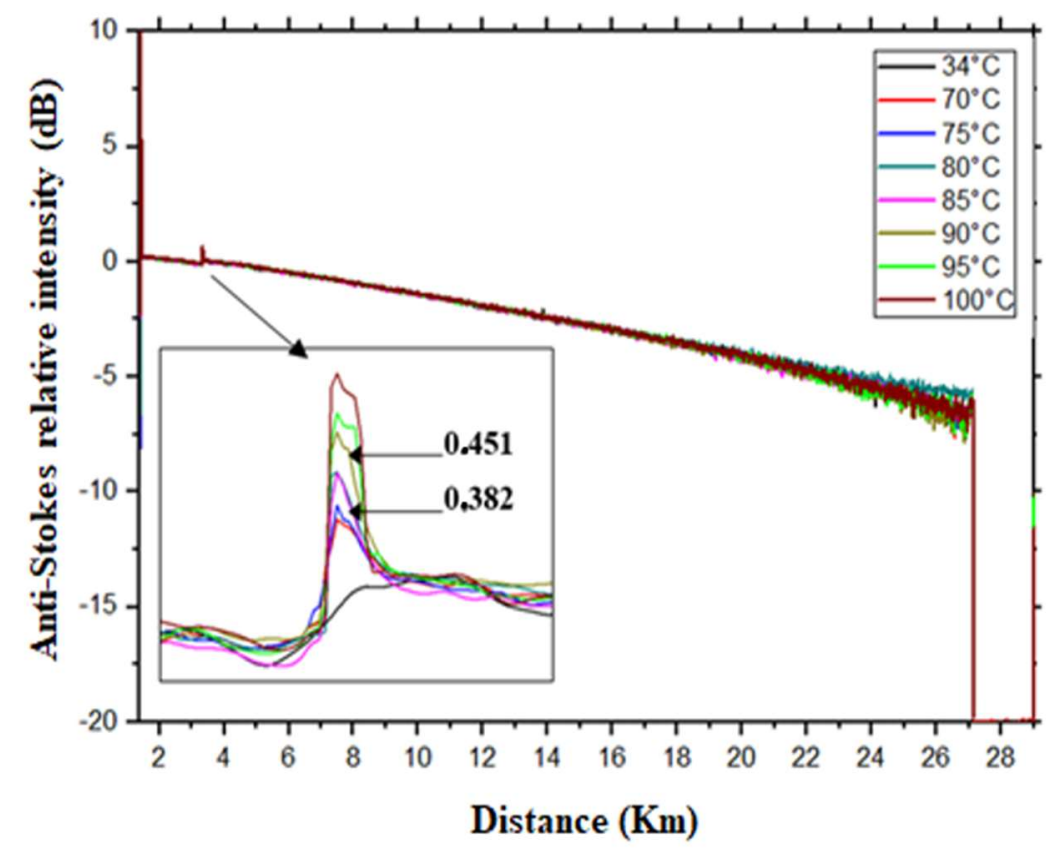

Fig.7. OTDR curves of the backscattered Raman anti-Stokes with Fiber-2 reel with $2 \mathrm{~m}$ of SMF fiber at the $3.4 \mathrm{~km}$ mark for $100 \mathrm{~ns}$ OTDR pulses.

The measured spatial distribution of temperatures, for the $2 \mathrm{~m}$ fiber spool placed at the $3.4 \mathrm{~km}$ mark in the sensor link, with 100 ns OTDR pulses, are presented in Fig. 8. These results show the operation of the sensor with temperature resolution of $10^{\circ} \mathrm{C}$ and sensitivity of $0.0092 \mathrm{~dB} /{ }^{\circ} \mathrm{C} @ 100 \mathrm{~ns}$. Even though the sensor sensitivity has been reduced as a result of the shorter length of fiber under heat, in comparison with the same system with longer spatial heat region (see Fig. 4-(a)), the results indicate that sensor range did not change, remaining at approximately $15 \mathrm{~km}$.

The parameters of the calibration lines provide quantitative information on sensor sensitivity for the tested configurations (combinations of OTDR pulse width and detection region). The analysis of these parameters allows for the conclusion that sensor sensitivity is higher when the OTDR operates with narrower pulse widths, in order to detect temperature variations in the first kilometers of the optical sensor link, and with better temperature resolution. On the other hand, when there are temperature fluctuations towards the end sections of the sensor link, the use of wider OTDR pulses result in higher 
sensitivity in the monitored region and smaller temperature resolution. The increase in sensitivity towards the final kilometers of the sensor link translates into an increase in sensor operating range.

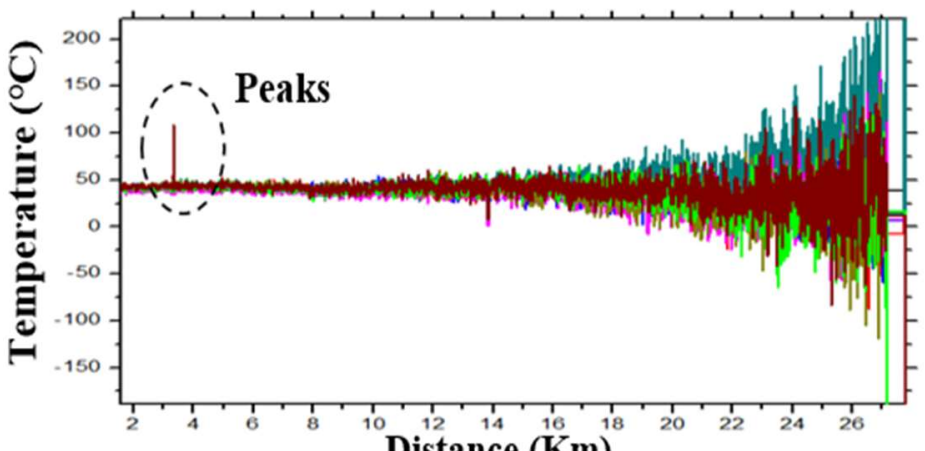

(a)

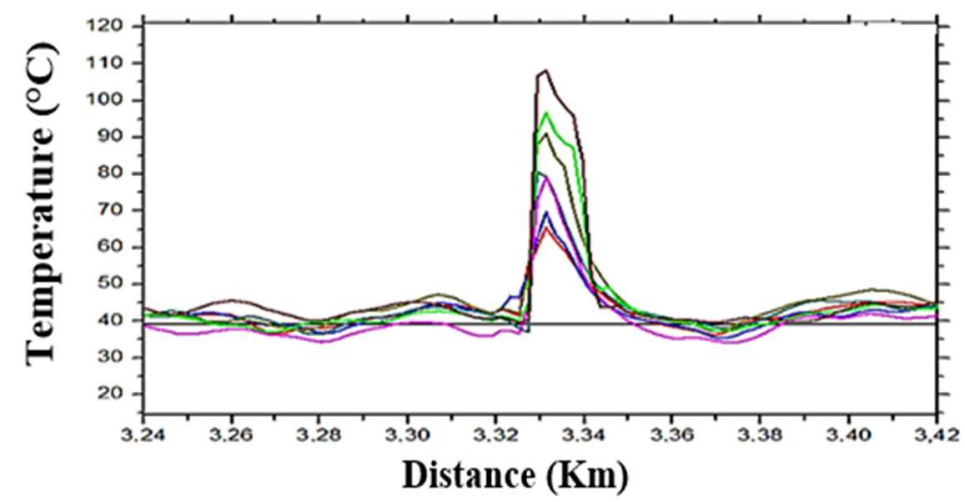

(b)

Fig. 8. Temperature distribution as a function of fiber length using $2 \mathrm{~m}$ of fiber heated at the $3.4 \mathrm{~km}$ mark, using OTDR pulses of $100 \mathrm{~ns}$ (a) and measured temperature spikes (b).

Fig. 9 shows the intensity of the anti-Stokes signal obtained by means of the theoretical model and the experimental setup, as a function of the heating temperature of Fiber-2 at the distance of $3.75 \mathrm{~km}$ employing OTDR pulses of $100 \mathrm{~ns}$. The points obtained via theoretical model illustrate the possibility of measurement with the sensor at higher temperatures (above $100^{\circ} \mathrm{C}$ ) and lower temperatures (below $0{ }^{\circ} \mathrm{C}$ ) than in the experiments produced here. In this case, sensor sensitivity is of $0.02685 \mathrm{~dB} /{ }^{\circ} \mathrm{C}$, a value not far from that obtained experimentally $\left(0.0169 \mathrm{~dB} /{ }^{\circ} \mathrm{C}\right)$. Again, the discrepancy between these values are due to the simplicity of the theoretical model. Observe that, at the temperature range measured experimentally $\left(33-100{ }^{\circ} \mathrm{C}\right)$, both the experimental and theoretical points feature linear behavior, which makes it possible to carry out an appropriate calibration of the system by a linear fitting. This would not be possible for a higher temperature range, as the theoretical points show. With that being said, the theoretical model is of interest, since it allows for an evaluation of the sensor under various perspectives and larger operating range. A summary of these results is shown on Table III. 


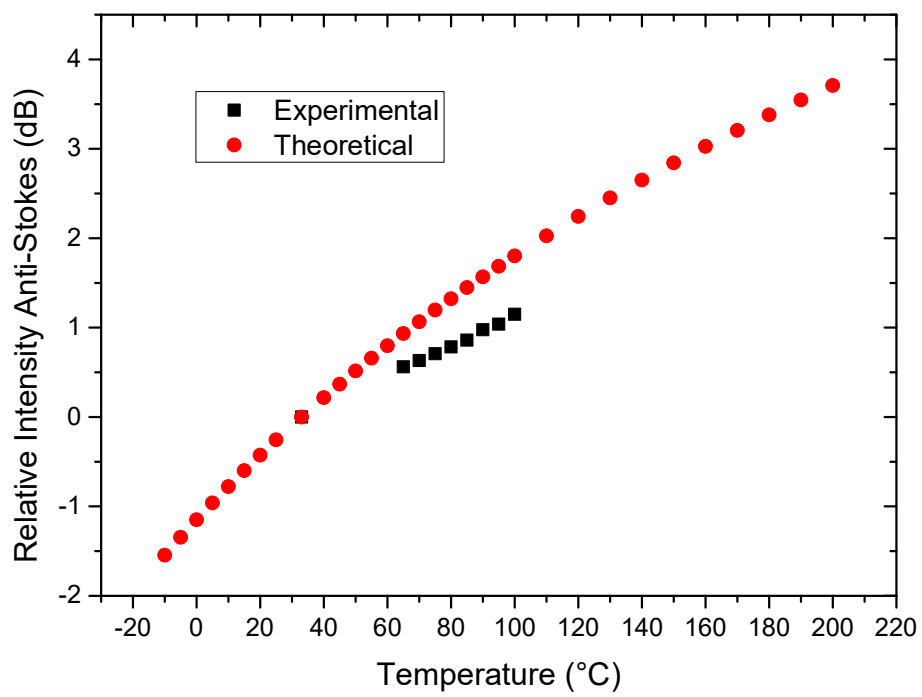

Fig. 9 - Experimental and theoretical points for the relative intensity anti-Stokes signal as a function of temperature, for $627 \mathrm{~m}$ of fiber in the $3.75 \mathrm{~km}$ mark under heat variations, for OTDR pulses of $100 \mathrm{~ns}$.

TABLE III. RESUlTS SUMMARY

\begin{tabular}{|c|c|c|c|c|c|}
\hline Fiber-2 Position & Fiber-2 length & Pulse width & Sensitivity & $\begin{array}{c}\text { Temperature } \\
\text { Resolution }\end{array}$ & Accuracy \\
\hline$[\mathrm{km}]$ & {$[\mathbf{m}]$} & [ns] & {$\left[\mathrm{dB} /{ }^{\circ} \mathrm{C}\right]$} & {$\left[{ }^{\circ} \mathbf{C}\right]$} & {$[\%]$} \\
\hline 3.4 & 627 & 100 & 0.0169 & 5 & 99.8 \\
\hline 3.4 & 627 & 100 & $0.02685^{*}$ & - & - \\
\hline 3.4 & 627 & 4000 & 0.0134 & 5 & 98.4 \\
\hline 25.4 & 627 & 4000 & 0.0075 & 10 & 99.4 \\
\hline 25.4 & 627 & 2000 & 0.0002 & - & 4.0 \\
\hline 25.4 & 627 & 1000 & 0.0001 & - & 3.3 \\
\hline 25.4 & 627 & 100 & 0.00001 & - & 12.0 \\
\hline 3.4 & 2 & 100 & 0.0092 & 10 & 93.3 \\
\hline
\end{tabular}

*theoretical

\section{CONCLUSIONS}

We presented the operation of a spontaneous Raman backscattering-based distributed fiberoptic temperature sensor (R-DTS) with a commercial OTDR and a standard EDFA, along with a theoretical model for the same sensor. We offered an innovative analysis of the sensor's sensitivity for seven different configurations, which combine 100 ns to 4000 ns OTDR pulses and different heating regions in a $27 \mathrm{~km}$ optical fiber link. The experimental results showed that sensor sensitivity depends on OTDR pulse width for different detection regions. In practice, the R-DTS may operate with two Brazilian Microwave and Optoelectronics Society-SBMO received 28 Sept 2018; for review 6 Nov 2018 ; accepted 20 Nov 2018 
pulse width values, using $100 \mathrm{~ns}$ pulses to detect temperature variations at the starting sections of the link, and $4000 \mathrm{~ns}$ pulses to monitor temperature towards the end portion of the link, with temperature resolutions of $5{ }^{\circ} \mathrm{C}$ and $10{ }^{\circ} \mathrm{C}$, respectively.

\section{ACKNOWLEDGMENT}

The authors thank CNPq, CAPES, FACEPE and the PPGEE of UFPE for the financial support.

\section{REFERENCES}

[1] G. Bolognini, and A. Hartog, "Raman-Based Fiber Sensors: Trends and Applications." Optical Fiber Technology, Elsevier, v. 19, n. 6, pp. 678-688, 2013.

[2] E. Udd and W. B. Spillman-Jr, Fiber Optic Sensors: An Introduction for Engineers and Scientists. John Wiley \& Sons, 2011.

[3] P. Jonghan, et al., "Raman-based distributed temperature sensor with simplex coding and link optimization." IEEE Photonics Technology Letters, v.18, n.17, pp. 1879-1881, 2006.

[4] J. Haus, Optical Sensors: Basics and Applications. Wiley Online Library, John Wiley \& Sons, 2010.

[5] F. Bassan et al, "Raman-Based Distributed Temperature Sensor Using Simplex Code and Gain Controlled Edfa". in: International Society for Optics and Photonics. 24th International Conference on Optical Fiber Sensors, v. 9634, pp. 96346, 2015.

[6] G. Bolognini, J. Park, P. Kim, D. Lee, F. Di Pasquale, and N. Park, "Performance Enhancement of Raman-Based Distributed Temperature Sensors Using Simplex Codes". In: IEEE. Optical Fiber Communication Conference, 2006 and the 2006 National Fiber Optic Engineers Conference. OFC 2006, pp. 3, 2006.

[7] M. Soto et al. "Distributed Temperature Sensor System Based on Raman Scattering Using Correlation-Codes." Electronics Letters, IET, v. 43, n. 16, pp. 862-864, 2007.

[8] F. Baronti et al., "Snr Enhancement of Raman-Based Long-Range Distributed Temperature Sensors Using Cyclic Simplex Codes." Electronics letters, IET, v. 46, n. 17, pp. 1221-1223, 2010.

[9] M. Tanner, S. Dyer, B. Baek, R. Hadfield, and S. Woo Nam, "High-Resolution Single-Mode Fiber-Optic Distributed Raman Sensor for Absolute Temperature Measurement Using Superconducting Nanowire Single-Photon Detectors.” Applied Physics Letters, AIP, v. 99, n. 20, pp. 201110, 2011.

[10] G. Bolognini, J. Park, M. Soto, N. Park, and F. Di Pasquale, "Analysis of Distributed Temperature Sensing Based on Raman Scattering Using Otdr Coding and Discrete Raman Amplification.” Measurement Science and Technology, IOP Publishing, v. 18, n. 10, pp. 3211, 2007.

[11] F. Bassan, F. Salgado, F. Fruett, and J. Rosolem, "Distributed Temperature Sensing System Using a Commercial OTDR and a Standard EDFA With Controlled Gain". in: International Society For Optics And Photonics. Fiber Optic Sensors and Applications XIII. v. 9852, pp. 98520S, 2016.

[12] J. Dakin, D. Pratt, G. Bibby, and J. Rose, "Distributed Antistokes Ratio Thermometry". in: Optical Society of America. Optical Fiber Sensors. a. p. PDS3, 1985.

[13] A. Hartog, A. Leach, and M, Gold, "Distributed Temperature Sensing in Solid-Core Fibre". Electronics letters, IET, v. 21, n. 23, pp. 1061-1062, 1985.

[14] J. Park, G. Bolognini, D. Lee, P. Kim, P. Cho, F. Di Pasquale, and N. Park, "Raman-Based Distributed Temperature Sensor with Simplex Coding and Link Optimization". IEEE photonics technology letters, IEEE, v. 18, n. 17, pp. 1879$1881,2006$.

[15] A. Signorini et al., "40 km long-range Raman-based distributed temperature sensor with meter-scale spatial resolution." In: Optical Fiber Communication (OFC), Collocated National Fiber Optic Engineers Conference, 2010 Conference on (OFC/NFOEC). IEEE, pp. 1-3, 2010.

[16] D. Hwang, D. Yoon, I. Kwon, D. Seo, and Y. Chung, "Novel Auto-Correction Method in a Fiber-Optic DistributedTemperature Sensor Using Reflected Anti-Stokes Raman Scattering.” Optics express, v. 18, n. 10, pp. 9747-9754, 2010.

[17] M. Soto et al., "Raman-based distributed temperature sensor with $1 \mathrm{~m}$ spatial resolution over $26 \mathrm{~km}$ SMF using lowrepetition-rate cyclic pulse coding." Optics letters, v. 36, n. 13, pp. 2557-2559, 2011.

[18] I. S. M. Shatarah, R. Olbrycht and B. Wiecek. Modeling of Spontaneous Raman Scattering in silica light guides for Distributed Temperature Sensing. In: 14 ${ }^{\text {th }}$ Quantitative InfraRed Thermography Conference. Berlin, Germany, 2018.

[19] M. A. Farahani and T. Gogolla. "Spontaneous Raman scattering in optical fibers with modulated probe light for distributed temperature Raman remote sensing', Journal of Lightwave Technology, v. 17, n. 8, pp. 1379-1391, 1999. 